\title{
African Buffalo Optimization Algorithm for Tuning Parameters of a PID Controller in Automatic Voltage Regulators
}

\author{
Julius Beneoluchi Odili \\ FSKKP \\ odili_julest@yahoo.com
}

\author{
Mohd Nizam Mohmad Kahar \\ Faculty of Computer Systems and \\ Software Engineering \\ mnizam@ump.edu.my \\ Universiti Malaysia Pahang \\ Kuantan 26300, Malaysia.
}

\author{
A. Noraziah \\ IBM Centre of Excellence \\ noraziah@ump.edu.my
}

\begin{abstract}
In this paper, we present the African Buffalo Optimization (ABO) approach for the metaheuristic tuning of the Proportional, Integral \& Derivative (PID) Controller parameters of Automatic Voltage Regulators in electrical power generation and transmission. ABO simulates the movement of African Buffalos as they migrate from place to place all over Africa in search of grazing pastures. The buffalos use just two basic vocalizations, namely the waaa and the maaa to organize the entire herd in their migration and search for food and water. Though a recently-designed algorithm, the ABO has been effective and efficient in solving a number of search optimization problems. The highpoints of the ABO include its use of few parameters, constant interactions among the buffalos and the deployment of the exploration and exploitations mechanisms of the algorithm in every iteration. The simulation outcome of the application of ABO to tune the parameters of a PID-Controller parameters of Automatic Voltage Regulators has been very competitive when compared similar outcomes of other metaheuristics tuners: BFO-PID, PSO-PID, GAPID, PID-PSO, PID Tuner and ACO-PID.
\end{abstract}

Keywords - Automatic Voltage Regulator, African Buffalo Optimization, metaheuristics, Proportional, Integral and Derivative, Tuning.

\section{INTRODUCTION}

To say that optimization is the heartbeat of the greatest scientific and technological achievements that mankind has experienced since the early $19^{\text {th }}$ century till date, may not be an overstatement. Optimization, basically, can be defined as the economics of technology, engineering and science since optimization emphasizes the need to obtain optimal outcomes with minimal input. Because of its strategic importance, optimization can be applied to virtually every aspect of scientific and engineering designs, commercial decision-making, production, business management, strategic planning, medicine, pharmacy, bio-medical engineering, bio-technology etc.

The popularity of engineering optimization has led to the design of several optimization algorithms such as the Particle Swarm Optimization [1], Genetic Algorithm [2], African Buffalo Optimization [3, 4] Simulated Annealing (SA) [5], Ant Colony Optimization [6], etc. These algorithm have been applied to different areas of science, technology and engineering such as job scheduling [7] Travelling Salesman's Problems [8, 9], computer networking [10], numerical function optimization [11], administration [12], tuning the Proportional, Integral and Derivative (PID) Controller's parameters of Automatic Voltage Regulars (AVR) [13] etc.

With special regards to optimizing the parameters of a PID Controller, the Ziegler and Cohen's technique is one of the first ever successful methods [14]. The successful application of this tuning method was celebrated for a number of years and this attacted the attention of researchers to investigate the application of metaheuristics in tuning the PID parameters of an Automatic Voltage Regulator, Dc Motors etc. Careful investiagation of the Ziegler and Cohen's tuning method reveals that it requires that the process models should first be minimised in order to make the system less complex [15]. Further investgations revealed that whenever this concern is not adequately addressed, the tuning outcome is not satisfactory thus resulting in system steady-state errors, system-delays, overshoot and eventual system instability. Whenever this unsatisfory tuning process occurs, the major objectives of a PID controller in the tuning process is defeated.

In addition, further research investigations revealed that the Ziegler and Cohen's technique requires a lot of professional expertise to obtain satisfactory tuning, even though it does not guarantee good performance at all times, in spite of the technical and professional input. This particular deficiency is one of the main motivations for researchers to investigate the possible use of metaheuristics for tuning PID parameters. Today, rigorous research efforts have led to the development of, Particle-Swarm Optimization PID (PSO-PID) [16], Genetic Algorithm PID ,(GA-PID) [17], Ant Colony Optimization PID (ACO-PID) [18], Bacteria-Foraging Optimization PID (BFO-PID) [19], PID-Tuner [20], African Buffalo Optimization (ABO-PID) [13] etc. The primary advantage of metaheuristic tuning of PID Conroller paramters in systems engineering ia as a result of their simplicity of operation, ease of use, ease of maintenance, dynamism, ease of implementation, effectiveness, low cost and efficiency . 
The rest of this paper is otganzed thus: section two elaborates on the PID; section three discusses the African Buffalo Optimization and its specific application to tuning the PID paramters of an AVR; section four highlights the experiemental results and section five drwas conclusions on the study. Next is acknowledgemnt of support for the study followed by the references.

\section{PID CONTROLLER}

The PID Controller is an example of a control loop feedback technique that is used in engineering, industrial and process control systems. The PID works optimally in electrical, electronic or electromechanical systems that has precise and distinguishable mathematical model. The PID Controller, basically, calculates three separate system parameters: proportional, integral and derivative coefficients. The proportional component of PID computes the value of the very current error. Similarly, the integral component calculates the result of the sum of recent errors while the derivative component is involved with the determination of the system's reaction based on the rate dynamism of the errors. The weighted sum of the functions of the three PID components is then imported into the control system to regulate the AVR. Some of the different AVR models with input PID controllers are presented in Figures 1-4.

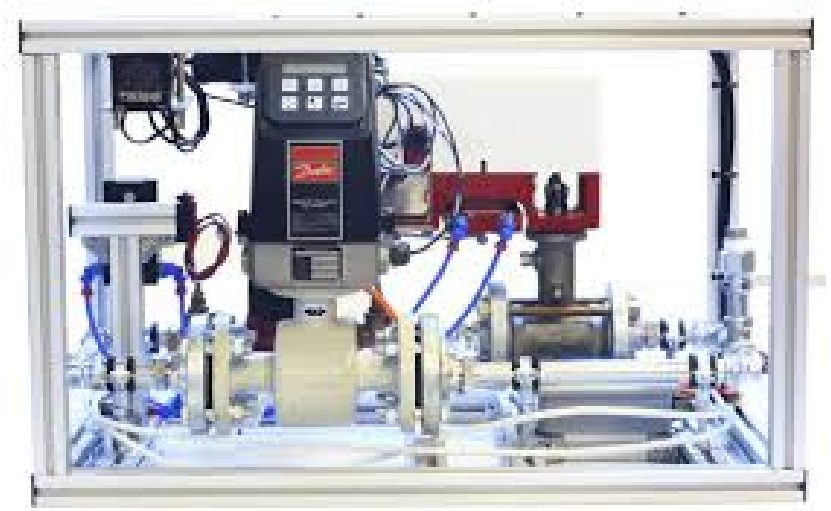

Figure 1: Picture of PID Controller type [21]

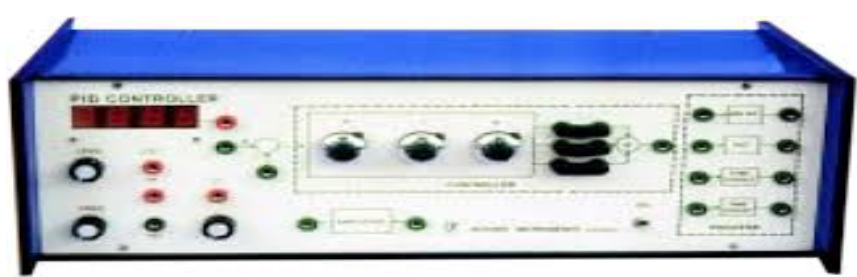

Figure 2: Picture of another brand of PID Controller

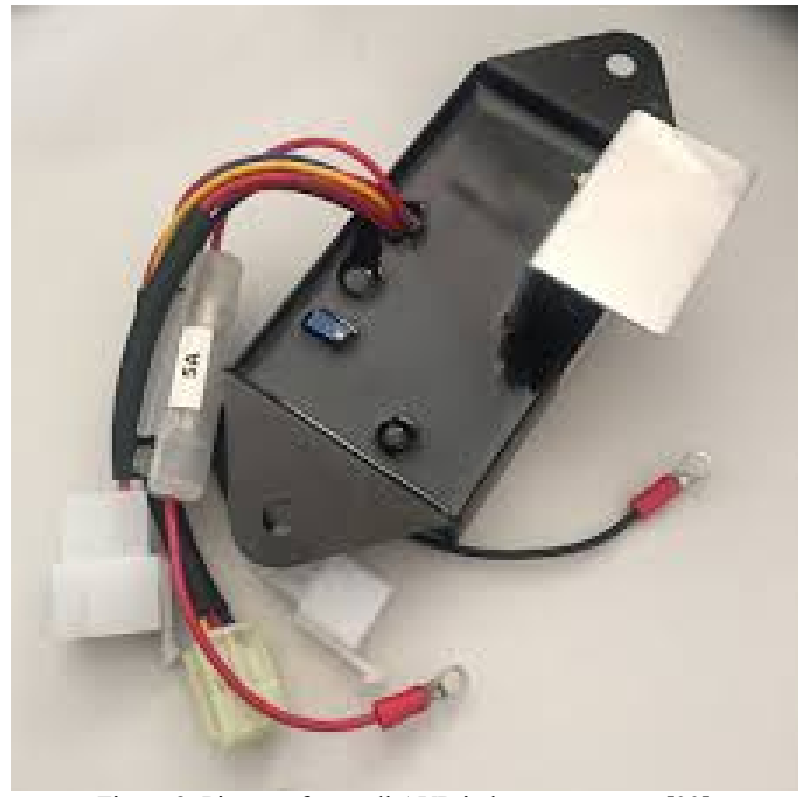

Figure 3: Picture of a small AVR in home generator [22]

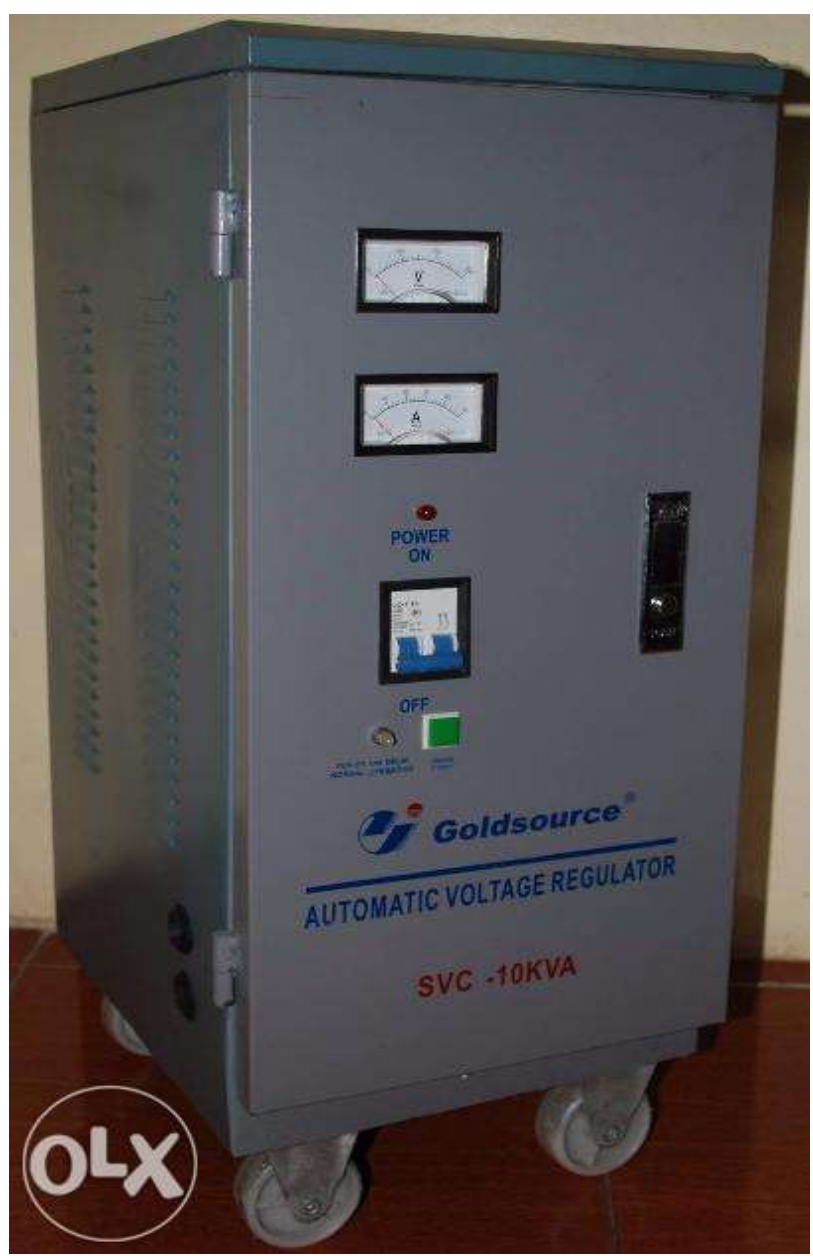

Figure 4: A medium size AVR[23] 


\section{A. Effects of PID on an AVR}

The primary duty of an AVR in electrical engineering is to regulate the entire electric power system, thus enhancing the stability of the power output system. The PID controller could be termed the heartbeat of the AVR since it is the PID that controls the dynamic response of the AVR ensuring the reduction or complete elimination of steady-state error.

The PID comprises of three principal parts: Proportional, Integral and Derivative components. The primary duty of the Proportional component $\left(\boldsymbol{G}_{p}\right)$ in a PID Controller reduces the rise time of the power generating system. Moreover, the Proportional component helps to minimize the steady state error but lacks the capacity to completely eliminate the steady-state error. On its part, the integral component $\left(G_{\mathrm{i}}\right)$ minimizes or in some cases totally eliminates the steady-state error. The integral component also helps in reducing the unpredictable response of the power generating system, thus enhancing the stability and reliability of the system. On the other hand, the primary function of the derivative control $\left(G_{d}\right)$ component is to enhance the system stability by reducing or totally eliminating the system's overshoot. Thus, the derivative component assists in improving the transient response of the power system. In summary, the cumulative effect of the PID Controller on a closed-loop electrical or electronic system is graphically presented in Table 1 . (Please note, however, that, in some special circumstances, there could be some minor changes).

TABLE 1: EFFECTS OF A PID ON A CLOSED LOOP SYSTEM

\begin{tabular}{|c|c|c|c|c|}
\hline Parameters & S-S error & Rise time & Over-shoot & Settling time \\
\hline $\boldsymbol{G}_{\boldsymbol{p}}$ & Decrease & Decrease & Increase & $\begin{array}{c}\text { Small } \\
\text { Change }\end{array}$ \\
\hline $\boldsymbol{G}_{\boldsymbol{i}}$ & Eliminate & Decrease & Increase & Increase \\
\hline $\boldsymbol{G}_{\boldsymbol{d}}$ & No Change & $\begin{array}{c}\text { Small } \\
\text { Change }\end{array}$ & Decrease & Decrease \\
\hline
\end{tabular}

\section{AFRICAN BUFFALO OPTIMIZATION \& PID TUNING}

The African Buffalo Optimization which is a recently developed metaheuristic algorithm was inspired by the herd management and organizational prowess of the African buffalos in their migrant lifestyle in African diverse landscapes (ranging from arid deserts, savannah and forests) in search of lush grazing fields to satisfy their huge appetites and sustain their kind in the ecosystem [24]. Using the /waaa/ (explore) and the /maaa/ (exploit) calls, the African buffalos are able to optimize their search for good grazing fields out of starving locations into very satisfying ones [25]. The African Buffalo Optimization (ABO) algorithm is presented in Figure 5 [26].

Step 1 Randomly initialize the buffalos to different location. Step2 Update the exploitation fitness of the buffalos using

$$
m_{k}{ }^{f}=m_{k}+l p 1\left(b g-m_{k}\right)+l p 2\left(b, k-w_{k}\right)
$$

Step 3 Update the buffalos' location using:

$$
w_{k}{ }^{t}=\frac{\left[w_{k}+w_{k}\right]}{r}
$$

Step 4 Verify if bgmax is updating? Yes, go to 6. If No after 10 iterations, return to Step 1

Step 5 Is the stopping criteria reached? No, go back to algorithm step 2, else proceed to Step 6

Step 6 Output best solution.

Figure 5: ABO Algorithm

\section{A. $A B O$ for $P I D$}

In evaluating the ABO-PID, an AVR system with ABOPID was implemented. Please recall that the main assignment of an AVR is to ensure a predetermined voltage output. As such, to regulate a voltage output requires a feedforward system as we have in 'open loop' systems [27] or a closed loop mechanism as in 'feedback' control systems [28]. Please note that a feedback control system is designed to send back some feedback information on the voltage output so as to ensure the predetermined output voltage is same as originally designed. To do this, the AVR may use an electromechanical component or an electronic device that has capacity to regulate one or more AC or DC voltages.

The components of an AVR includes the exciter, generator, amplifier, and sensor: all working together to ensure the output voltage stability in the power engineering. It is important to note that in virtually every power system, increasing the load usually leads to a drop in the output voltage, hence the need for a PID. A PID controller is, therefore, helps to minimize the output error arising increase load by ensuring improved dynamic response. Needless to say, therefore, that the stability or otherwise of the AVR seriously affects the stability and security of the entire power system. The Block diagram of an ABO-PID is presented in Figure 5. 


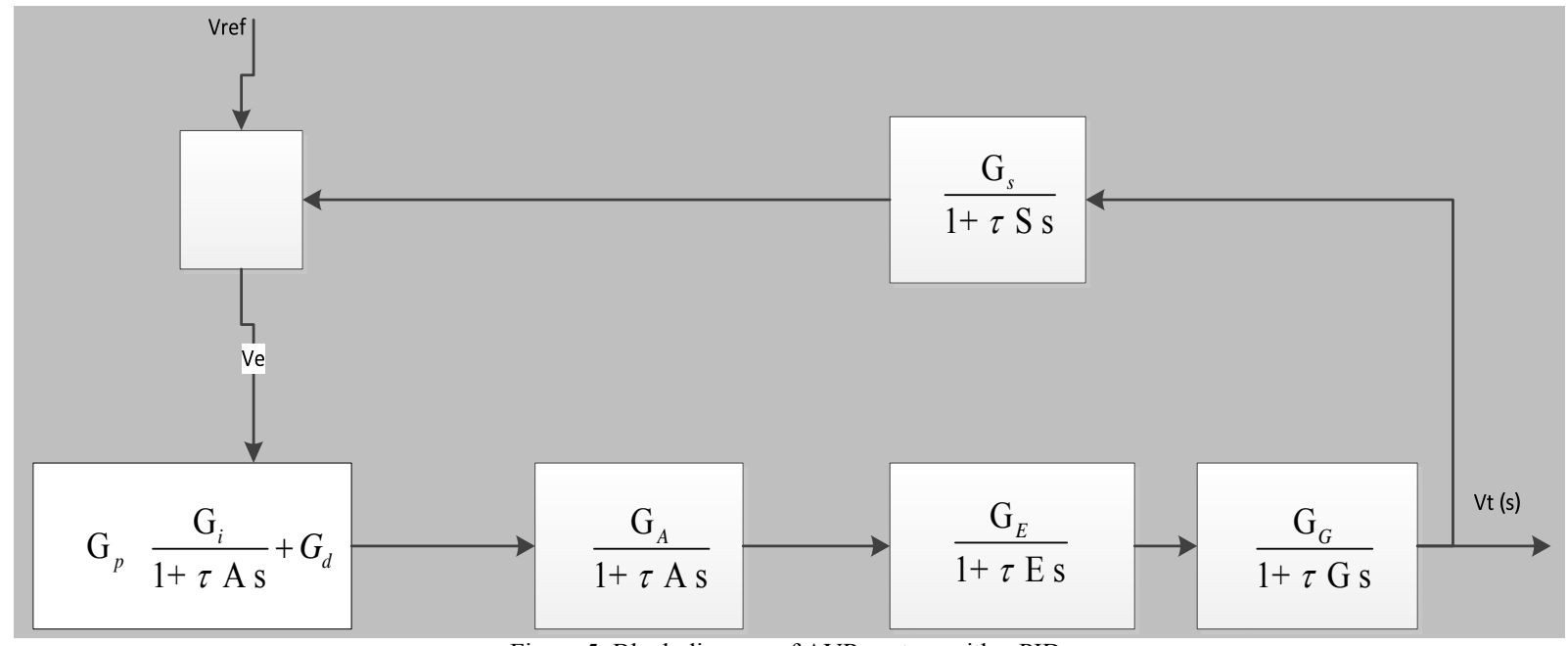

Figure 5: Block diagram of AVR system with a PID.

The linear cum mathematical representation of an AVR in power generators is:

$$
\operatorname{Amplifier}\left(G_{A}\right)=V R(s) / V \in(s)=G_{A} / 1+\tau_{h} S
$$

where $G_{A}$ denotes the Amplifier gain; $\tau_{A}$ denotes the Time constant in $\mathrm{S}$ domain. Normal values of $G_{A}$ ranges from 10 to 400 . Amplifier's time constant is typically very small and ranges from $0.02 \mathrm{~s}$ to $0.1 \mathrm{~s}$.

The transfer function (TF) of an Exciter could be represented by a gain $G_{\vec{E}}$ with a single time constant:

$$
\text { Exciter }=\mathscr{F} F(S) / P R(S)=G_{E} / 1+\tau_{E}(S)
$$

In this instance, $\mathrm{K}_{\mathrm{E}}$ denotes Exciter gain; $\tau_{\mathcal{E}}(S)$ the Time constant in $S$ domain. Usual values of $G_{E}$ ranges from 10 to 400. The time constant ranges from $0.5 \mathrm{~s}$ to $1.0 \mathrm{~s}$.

Furthermore, the transfer function that relates the generator terminal voltage could be represented by a gain $G_{G}$ and time constant s:

$$
\text { Generator }=\operatorname{Vt}(S) / F f(S)=G_{Q} / 1+\tau_{Q}(S)
$$

Please note that the values of the constants depend on the generator load. $G_{G}$ and could range from between 0.7 and 1.0 , or between 1.0 and $2.0 \mathrm{~s}$ depending on when the generator has full load to when it has no load.

Lastly, a simple first-order representation of the transfer function of a sensor is:

$$
\text { Sensor }=F Q(S) / P t(S)=G_{\Sigma} / 1+\tau_{S}(S)
$$

where $\tau_{s}$ is usually very small, and ranges from 0.001 to 0.06 s.

\section{B. ABO Strategy for Tuning the PID Parameters of An AVR}

The searching strategy of the ABO-PID Controller is is as follows:

Step 1: Randomly initialize the buffalo population in sets of three buffalos: meaning, when there are $\mathrm{N}$ buffalos, there exists $A / 3$ components. Simply set $s$ which denotes step function as 2

Step 2: Compute the buffalos' exploitation and exploration fitness using Equations 5 and 6 respectively

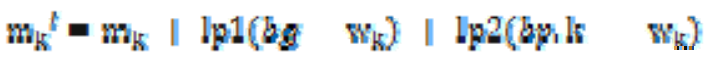

$$
\begin{aligned}
& \mathrm{w}_{\mathrm{k}}{ }^{t}=\frac{\left(w_{\mathrm{r}}+\mathrm{w}_{\mathrm{n}}\right)^{2}}{r}
\end{aligned}
$$

Step 3: Determine $G p G r$ and $G d$ for each set of buffalos

Step 4: Plot the $G p G$ and $G d$ into the PID benchmark transfer function denoted by:

$$
G p(s)+\frac{x}{s}+6 d(s)
$$

Select the set of buffalos with the best fitness value. Set as the $b_{g}$

Step 5: Compute $x / y$. If the outcome is 1 , denoting a steady state, terminate the run, otherwise, go back to Step 2

\section{EXPERIMENTS AND DISCUSSION OF RESULTS}

In this study, ABO-PID was implemented using MATLAB and executed on a MATLAB Compiler 2012b. Fitness function is the Integrated Absolute Squared Error. 
The parameters are presented in Table 2 and the experimental outcome is presented in Figure 6.

TABLE 2: PID TUNING PARAME
\begin{tabular}{|c|c|}
\hline Parameters & Values \\
\hline $\boldsymbol{G}_{\boldsymbol{A}}$ & 10 \\
\hline $\boldsymbol{G}_{\boldsymbol{E}}$ & 1.0 \\
\hline $\boldsymbol{G}_{\boldsymbol{G}}$ & 1.0 \\
\hline $\boldsymbol{G}_{\boldsymbol{S}}$ & 1.0 \\
\hline $\boldsymbol{\tau}_{\boldsymbol{A}}$ & 0.1 \\
\hline $\boldsymbol{\tau}_{\boldsymbol{E}}$ & 0.4 \\
\hline $\boldsymbol{\tau}_{\boldsymbol{G}}$ & 1.0 \\
\hline $\boldsymbol{\tau}_{\boldsymbol{S}}$ & 0.01 \\
\hline
\end{tabular}

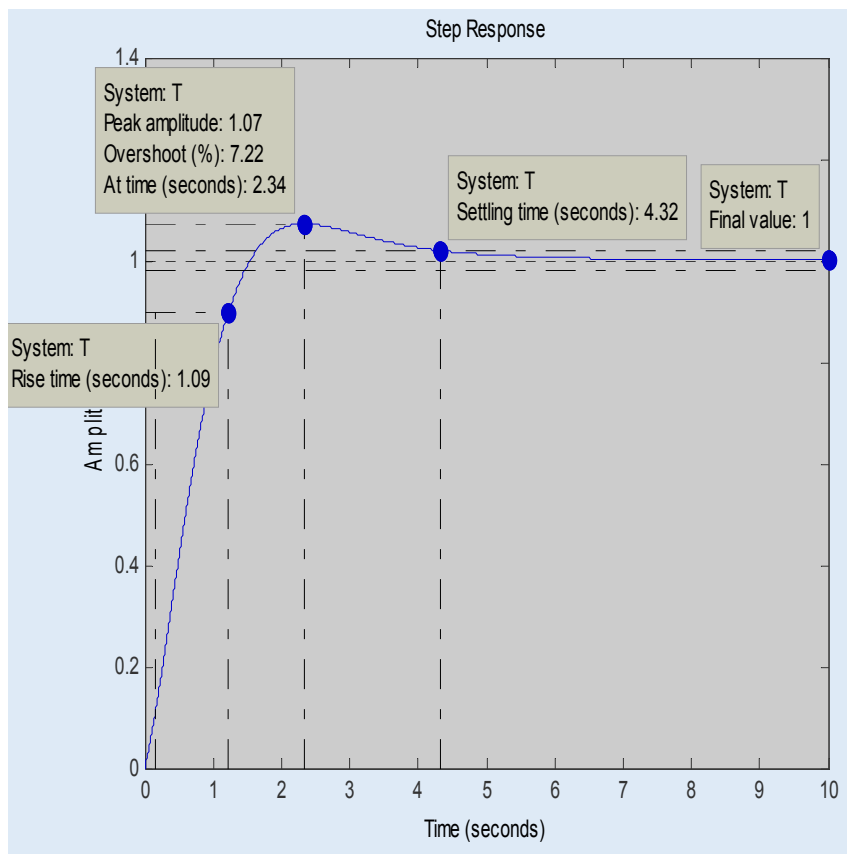

Figure 6: Time-domain output of ABO-PID
We note that the variable $V e$ in the Block diagram (Figure 3) can be obtained using:

$$
\nabla \theta=\nabla t(s)-\nabla r e f(s)
$$

Here, $V e$ denotes the tracking error. To obtain the tracking error, subtract the input voltage otherwise called reference signal ( $\nabla r e f(s))$ from the output $(\nabla t(s))$ signal. The obtained error voltage $(V \theta)$ signal is then fed-back to the PID controller whose assignment is to sum up PID of this voltage error.

In addition, please note that the PID controller's transfer function $(\mathrm{TF})$ is:

$$
G p+G i / s \frac{a}{s}+G d(s)
$$

Similarly, the transfer function of the AVR components is:

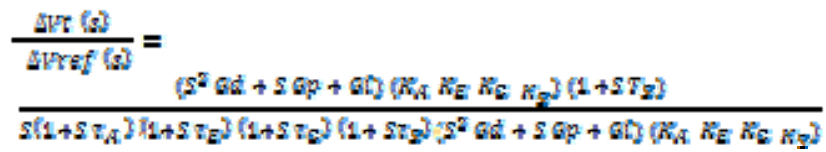

The experimental outcome is compared with those from other optimization search algorithms such as the GA-PID, PID-PSO, ACO-PID, BFOPID [29], PSO-PID, LQR-PID and PID-tuner tuner [30]. The simulation result is as presented below in Table 3 .

TABLE 3. PID TUNING RESULTS

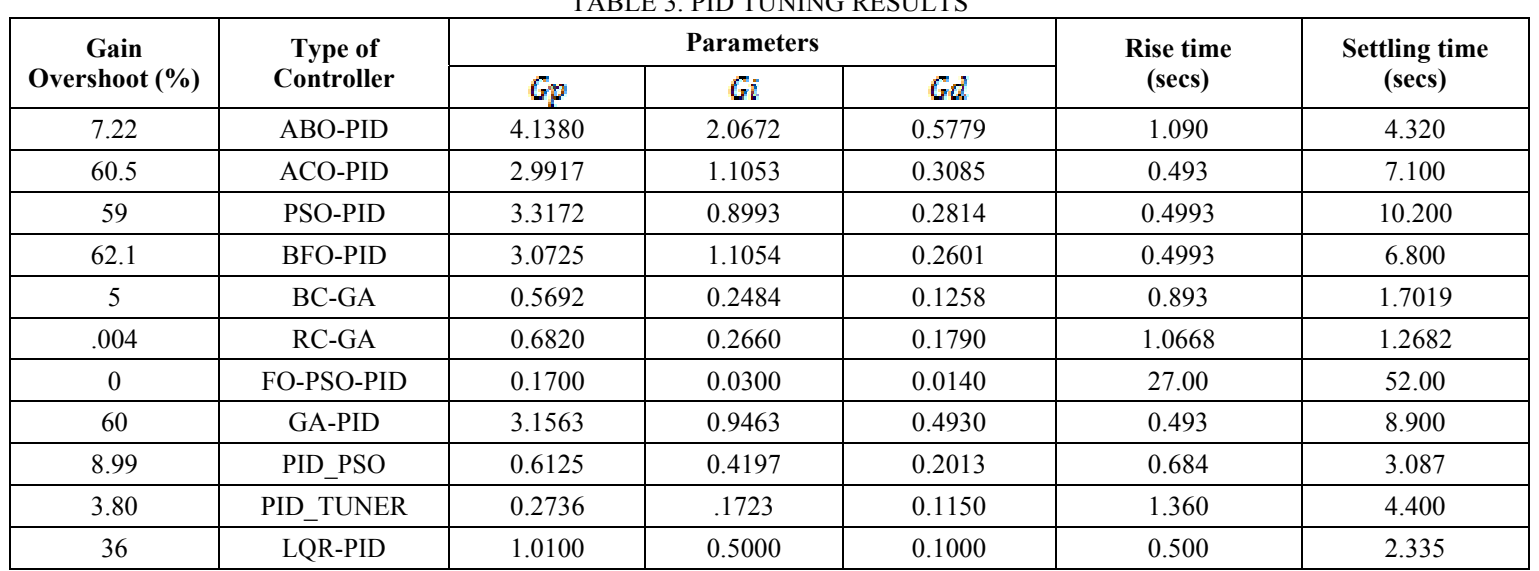


The data in Table 3 is quite intriguing as it tends to validate the No Free Lunch theorem of search optimization algorithms [31]. The No Free Lunch Theorem asserts that that there is nothing like the best search optimization algorithm and that an algorithm usually perform very well in some given problems but not as well in other problems. Further, it states that performance of an algorithm is primarily due to proper tuning of the algorithm's parameters for some given problems. In other words, what is of particular user/researcher determines his choice of algorithm. The No Free Lunch Theorem concludes that optimization search algorithms can perform excellently well in some given problems, post fair performance in some other optimization problems and perform poorly in the remaining cases [32].

Table 3, basically, highlights the merits and demerits of the algorithms under investigation, to such a point that a parameter of interest to a practitioner determines the algorithm to employ in tuning the PID parameters of an AVR. For instance, if the user is interested in having a system with the least percentage gain overshoot, then his choice will be will be FFO-PID since it has $0 \%$. The next choice would be Real-coded Genetic Algorithm (.004\%); PID-tuner (3.8\%); Binary-coded Genetic Algorithm (5\%); ABO-PID (7.22\%), PID-PSO (8.99\%), LQR-PID (36\%); PSO-PID (59\%), GA-PID (60\%), ACO-PID (60.5\%) and BFO-PID (62.1\%) respectively.

Similarly, if the researcher/user is interested in a system with very fast rise time of the power generating set, then GA-PID and ACO-PID are the algorithms of first choice since both algorithms have a rise time 0.493 second in comparison to PSO-PID and BFO-PID (0.4993 second); LQR-PID (0.5 second); PID-PSO, (0.684 second); BC-GA, (0.893 second); RC-GA (1.0668 second); ABO-PID, (1.09); PID-tuner, (1.36 seconds) and FO-PSO-PID (27 seconds).

Moreover, if the settling time parameter is the main consideration, then the 'best' algorithm is RC-GA since it has the least settling time of 1.2682 seconds). The next choice should be BC-GA with 1.7019 seconds, then LQRPID with 2.335 seconds, followed by 3.087 seconds for PID-PSO, 4.320 seconds (ABO-PID), 4.4 seconds (PIDtuner), 6.8 seconds (BFO-PID), 7.1 seconds (ACO-PID), 8.9 seconds (GA-PID), 10.2 seconds (PSO-PID) and, then, 52 seconds (FO-PSO-PID).

\section{CONCLUSION}

From the foregoing discussion and analysis, it may be safe to conclude that a good metaheuristic tuner of a PID Controller of an AVR is one that provides a fair balance in optimizing the different parameters in its effort at obtaining efficient and effective tuning. Moreover, the performance of FO-PID is worthy of special mention. Though, the FO-PID posted no gain overshoot yet it had the slowest rise time and setting time. In other words, FO-PID sacrificed the rise and settling times in order to obtain $0 \%$ gain overshoot. This certainly is not a mark of an effective optimization algorithm [33]. On the other hand, the effectiveness of the ABO-PID can be seen in its capacity to ensure harmonious tuning through a good trade-off of the tuning parameters thereby maintaining efficient and harmonious stability of the AVR. The performance of the ABO-PID validates the findings of some researchers that a good optimization algorithm should be robust [33]. The robustness of an Algorithm refers to its capacity to provide competitive outcomes in a wide range of optimization problems.

Juxtaposing the competitive tuning capability of ABO in This study with its performance in solving benchmark travelling salesman problems [34] and numerical optimization test functions [25], it may be safe to conclude that the $\mathrm{ABO}$ is a good addition to the existing body of bioinspired techniques.

\section{ACKNOWLEDGMENT}

The authors are grateful to the Faculty of Computer Systems and Software Engineering, Universiti Malaysia Pahang, Kuantan 26300, Malaysia for providing funding for this study through Grant GRS 1403118. Similarly, the additional funding of the Fundamental Research Grant Scheme under Grant RDU140101 is highly appreciated.

\section{REFERENCES}

[1] M. A. Kunna, T. A. A. Kadir, A. S. Jaber, and J. B. Odili, "LargeScale Kinetic Parameter Identification of Metabolic Network Model of E. coli Using PSO," Advances in Bioscience and Biotechnology, vol. 6, p. $120,2015$.

[2] M. H. Moradi and M. Abedini, "A combination of genetic algorithm and particle swarm optimization for optimal DG location and sizing in distribution systems," International Journal of Electrical Power \& Energy Systems, vol. 34, pp. 66-74, 2012.

[3] M. N. M. K. Julius Beneoluchi Odili*, Shahid Anwar, "African Buffalo Optimization: A Swarm-Intelligence Technique," in 2015 IEEE International Symposium on Robotics and Intelligent Sensors (IRIS2015), 2015, p. 7.

[4] J. B. Odili and M. N. Mohmad Kahar, "Solving the Traveling Salesman's Problem Using the African Buffalo Optimization," Computational intelligence and neuroscience, vol. 2016, pp. 1-12, 2016.

[5] K. A. Dowsland and J. M. Thompson, "Simulated annealing," in Handbook of Natural Computing, ed: Springer, 2012, pp. 1623-1655.

[6] J. B. Odili, "Application of Ant Colony Optimization to Solving the Traveling Salesman's Problem," Science Journal of Electrical \& Electronic Engineering, vol. 2013, 2013.

[7] B. Xu, C. Zhao, E. Hu, and B. Hu, "Job scheduling algorithm based on Berger model in cloud environment," Advances in Engineering Software, vol. 42, pp. 419-425, 2011.

[8] J. B. Odili and M. N. M. Kahar, "Solving the Traveling Salesman's Problem using the African Buffalo Optimization."

[9] J. B. Odili, M. N. M. Kahar, S. Anwar, and M. A. K. Azrag, "A comparative study of African Buffalo Optimization and Randomized Insertion Algorithm for asymmetric Travelling Salesman's Problem," in Software Engineering and Computer Systems (ICSECS), 2015 4th International Conference on, 2015, pp. 90-95.

[10] S. Anwar, J. M. Zain, M. F. Zolkipli, Z. Inayat, A. N. Jabir, and J. B. Odili, "Response option for attacks detected by intrusion detection system," in Software Engineering and Computer Systems (ICSECS), 2015 4th International Conference on, 2015, pp. 195-200.

[11] J. B. Odili and M. N. M. Kahar, "African Buffalo Optimization (ABO): a New Meta-Heuristic Algorithm." 
[12] J. B. Odili, "Staff development programs and job performance: Implications for productivity in Lagos state ministry of education ", 2013, pp. 330-334.

[13] J. B. Odili, M. Nizam, and M. Kahar, "African Buffalo Optimization Approach to the Design of PID Controller in Automatic Voltage Regulator System," National Conference for Postgraduate Research, Universiti Malaysia Pahang, vol. September, 2016, pp. 641-648, 2016.

[14] S. Das, A. Chakraborty, J. K. Ray, S. Bhattacharjee, and B. Neogi, "Study on Different Tuning Approach with Incorporation of Simulation Aspect for ZN (Ziegler-Nichols) Rules," International Journal of Scientific and Research Publications, vol. 2, pp. 1-5, 2012.

[15] M. Korkmaz, Ö. Aydoğdu, and H. Doğan, "Design and performance comparison of variable parameter nonlinear PID controller and genetic algorithm based PID controller," in Innovations in Intelligent Systems and Applications (INISTA), 2012 International Symposium on, 2012, pp. 1-5.

[16] M. I. Solihin, L. F. Tack, and M. L. Kean, "Tuning of PID controller using particle swarm optimization (PSO)," International Journal on Advanced Science, Engineering and Information Technology, vol. 1, pp. 458-461, 2011.

[17] M. J. Neath, A. K. Swain, U. K. Madawala, and D. J. Thrimawithana, "An optimal PID controller for a bidirectional inductive power transfer system using multiobjective genetic algorithm," Power Electronics, IEEE Transactions on, vol. 29, pp. 1523-1531, 2014.

[18] M. Ünal, A. Ak, V. Topuz, and H. Erdal, Optimization of PID controllers using ant colony and genetic algorithms vol. 449: Springer, 2012.

[19] I. B. Manuaba, M. Abdillah, A. Priyadi, and M. H. Purnomo, "COORDINATED TUNING OF PID-BASED PSS AND AVR USING BACTERIAL FORAGING-PSOTVAC-DE ALGORITHM," Control and Intelligent Systems, vol. 43, 2015.

[20] P. Aravind, M. Valluvan, and S. Ranganathan, "Modelling and Simulation of Non Linear Tank," International Journal of Advanced Research in Electrical, Electronics and Instrumentation Engineering, vol. 2, pp. 842-849, 2013.

[21] A. J. Al Gizi, M. Mustafa, K. M. Al Zaidi, and M. K. Al-Zaidi, "Integrated PLC-fuzzy PID Simulink implemented AVR system," International Journal of Electrical Power \& Energy Systems, vol. 69, pp. 313-326, 2015.

[22] Aliexpress, "Automatic Voltage Regulators 220volts," https://www.aliexpress.com/price/automatic-voltage-regulator220v price.html, vol. Accessed on 28/12/2016, 2016.

[23] Goldsource, "Automatic Voltage Regulators," http://goldsourceexport.en.china.cn/, vol. Accessed on 28/12/2016, 2016.

[24] J. B. Odili, M. N. M. Kahar, and S. Anwar, "African Buffalo Optimization: A Swarm-Intelligence Technique," Procedia Computer Science, vol. 76, pp. 443-448, 2015.

[25] J. B. Odili and M. N. M. Kahar, "Numerical Function Optimization Solutions Using the African Buffalo Optimization Algorithm (ABO)," British Journal of Mathematics \& Computer Science, vol. 10 , pp. 1-12, 2015.

[26] J. B. M. N. M. Odili, Kahar, "African Buffalo Optimization," International Journal of Software Engineering \& Computer Systems vol. 2, pp. 28-50, 2016.

[27] L. Angquist, A. Antonopoulos, D. Siemaszko, K. Ilves, M. Vasiladiotis, and H.-P. Nee, "Open-loop control of modular multilevel converters using estimation of stored energy," IEEE transactions on industry applications, vol. 47, pp. 2516-2524, 2011.

[28] Y.-J. Liu, S.-C. Tong, D. Wang, T.-S. Li, and C. P. Chen, "Adaptive neural output feedback controller design with reduced-order observer for a class of uncertain nonlinear SISO systems," IEEE Transactions on Neural Networks, vol. 22, pp. 1328-1334, 2011.

[29] J. B. O. a. M. N. M. Kahar, "African Buffalo Optimization," International Journal of Software Engineering \& Computer Systems, vol. 2, pp. 28-50, 2016.

[30] V. K. Bhatt and D. S. Bhongade, "Design Of PID Controller In Automatic Voltage Regulator (AVR) System Using PSO Technique," International Journal of Engineering Research and Applications (IJERA), vol. 3, pp. 1480-1485, 2013.

[31] H. Xu, C. Caramanis, and S. Mannor, "Sparse algorithms are not stable: A no-free-lunch theorem," Pattern Analysis and Machine Intelligence, IEEE Transactions on, vol. 34, pp. 187-193, 2012.

[32] D. Kifer and A. Machanavajjhala, "No free lunch in data privacy," in Proceedings of the 2011 ACM SIGMOD International Conference on Management of data, 2011, pp. 193-204.

[33] C. Khompatraporn, J. D. Pintér, and Z. B. Zabinsky, "Comparative assessment of algorithms and software for global optimization," Journal of Global Optimization, vol. 31, pp. 613-633, 2005.

[34] J. B. Odili, M. N. Kahar, and A. Noraziah, "Solving Traveling Salesman's Problem Using African Buffalo Optimization, Honey Bee Mating Optimization \& Lin-Kerninghan Algorithms," World Applied Sciences Journal, vol. 34, pp. 911-916, 2016. 